\title{
Tools for Trigger Aware Analyses in ATLAS
}

Attila Krasznahorkay on behalf of the ATLAS TDAQ Collaboration

New York University, USA; on leave from the Institute of Nuclear Research of the Hungarian Academy of Sciences

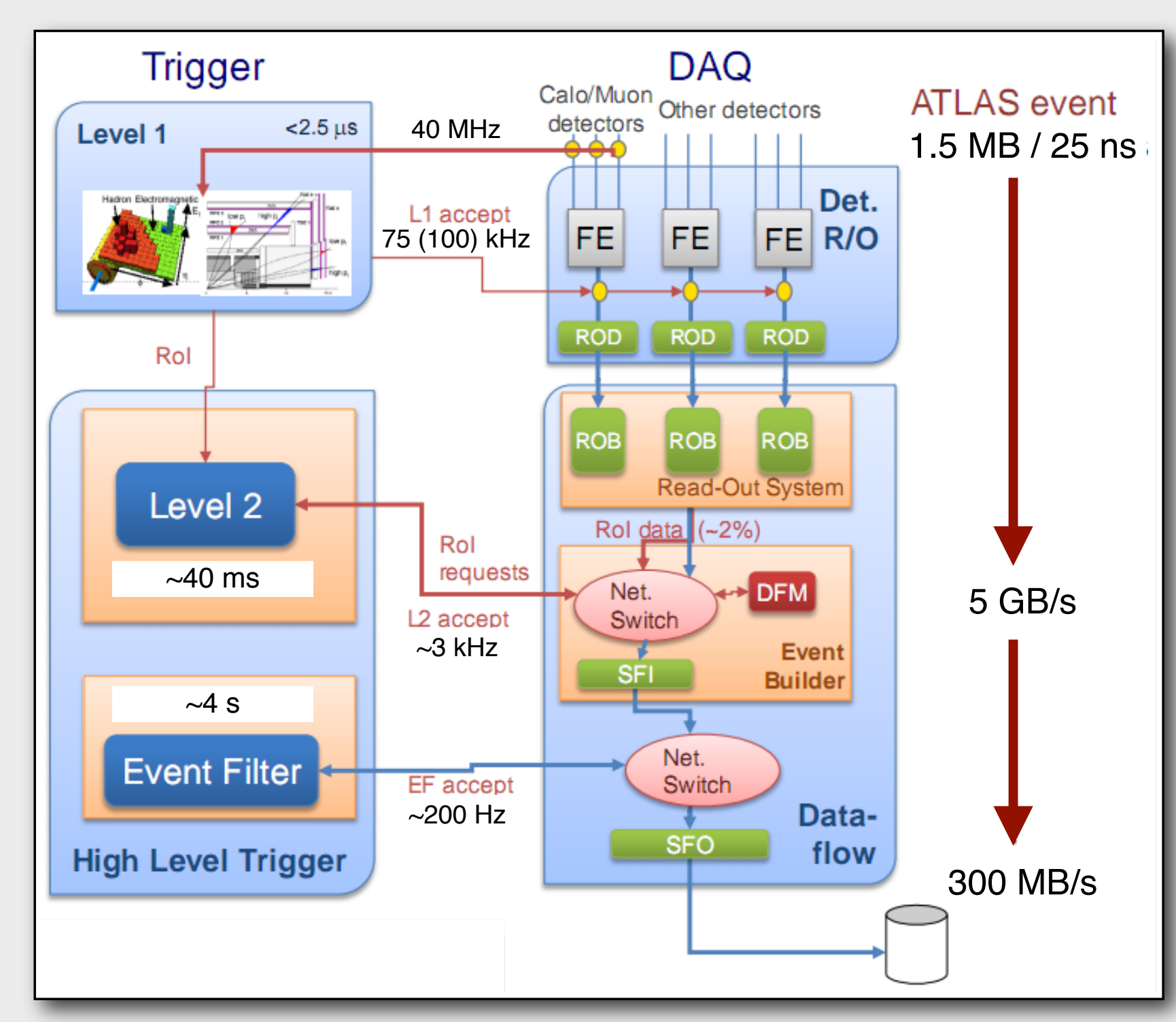

\section{The ATLAS trigger system}

ATLAS is one of the experiments at the Large Hadron Collider (LHC). It has a three-level trigger for selecting high-energy proton-proton (and heavy ion) collisions produced by the Large Hadron Collider for physics. At the nominal LHC luminosity the system will have to select 200 bunch crossings with proton-proton interactions out of about 40 million every second. The structure of the system is the following:

The first level of the trigger (Level 1) is built from custom hardware that identifies electromagnetic and hadronic activity in the calorimeters, and charged particles passing the muon spectrometer. It also identifies Regions of Interest (Rol) in the detector, so the later trigger levels do not have to scan the entire detector

The second level of the trigger (Level 2) uses PCs running special reconstruction algorithms which all collect data from a small region of the detector, flagged by the Level 1 trigger hardware

The last level of the trigger (Event Filter) also uses PCs, running mainly algorithms used in the offline event reconstruction, adapted to running in the High Level Trigger environment.

The High Level Trigger (Level 2 and Event Filter) [1] uses a common framework for making decisions about the events. The logic of the code execution in the High Level Trigger is shown on the right. The algorithms receive a seed from the previous trigger level, this way they can look at specific areas of the detector, minimizing data transfer, and speeding up execution. Two different algorithm types are defined:

Feature extraction (FEX) algorithms: These algorithms reconstruct the properties of physical objects (i.e. electromagnetic cluster or electron) on which further algorithms operate or apply selection

Hypothesis (Hypo) algorithms: These algorithms are responsible for making the trigger decision. They perform a selection of the objects reconstructed by the FEX algorithms. Depending on whether the reconstructed objects pass the quality cuts imposed by the hypothesis algorithm, the algorithm can mark the objects as either passing or not passing the given hypothesis.

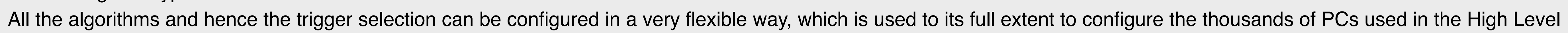
Trigger selection.

\section{The Trigger Analysis Tools group}

To simplify the task of analyzing the performance of the ATLAS trigger, and hide some of the complexities of the Level 1 and High Level Triggers, ATLAS has a small group working on implementing and maintaining tools related to the ATLAS trigger, used in data analysis. [2] The group is also responsible for giving tutorials about its tools. This poster summarizes some of the projects of the Trigger Analysis Tools group.

Full access to all the objects created by the trigger, separately for each trigger path

- Analysis objects created from the data provided by the Level 1 trigger

- Objects created by the High Level Trigger reconstruction algorithms, including "ancestral relations" between the objects.

Beside the "physics decision", which means that the trigger was not running in any

"special" mode, the tool allows experts to look at the performance of the triggers running with special configurations.

This tool is considered to be the only supported way to access the trigger decision information

in a physics analysis. It can be used both from the ATLAS offline software environment, and from stand-alone $\mathrm{C}++$ and Python environments.

\section{TrigDecisionTool}

A single $\mathrm{C}++$ tool working in the offline software of ATLAS, providing the following features:

Event-level trigger decisions: Analyzers can ask the tool if a trigger with a given name was fired in the event with various conditions

Access to the trigger configuration which was active when recording the given event. The configuration of the trigger system is provided through a simple interface which makes it easier to reproduce the configuration of the trigger of interest

\section{Code examples}

// Check if a given trigger fired in the current event:

trigDecTool->isPassed ( $E F \_m u 20 "$ );

// Retrieve all the objects reconstructed by this trigger

FeatureContainer features = trigDecTool->features ( "EF_mu20" )

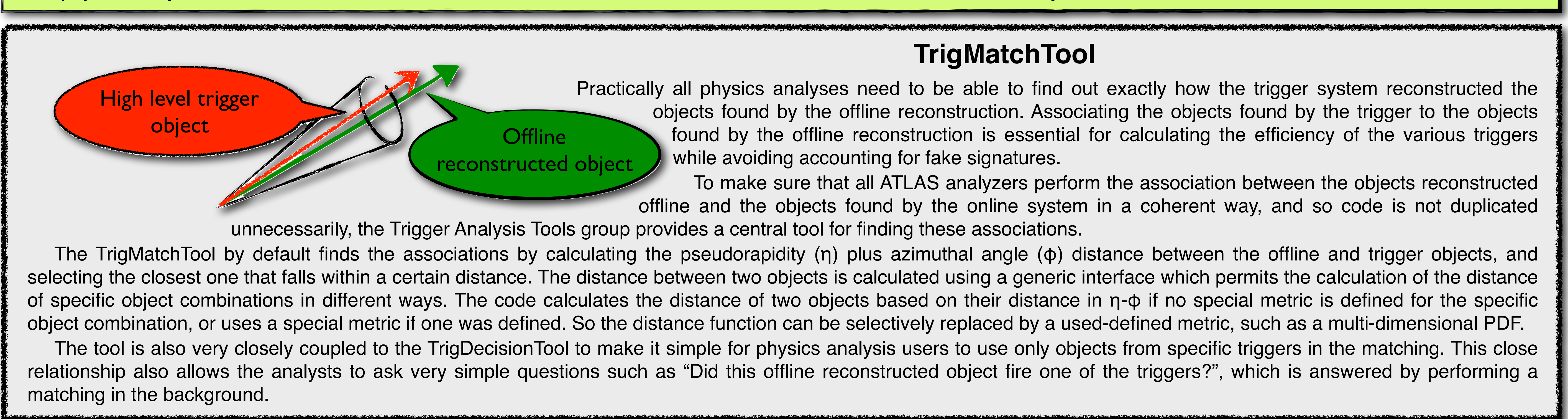

\section{InSituPerformance framework}

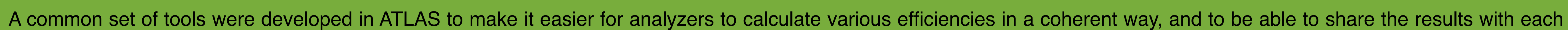
others through a common interface. The InSituPerformance code is basically composed of two separate parts:

A set of algorithms and tools that are developed to extract the efficiencies of the offline reconstruction and trigger algorithms for finding physical objects, directly from the recorded data.

A collection of data classes and helper code for saving these objects into files that can be shared amongst the groups. We define the efficiency as the fraction of events fulfilling some requirements over the number of all tested events. As a result we store the raw number of events before and after some "selection", possibly partitioned into separate regions of the phase space (for instance in transverse momentum and pseudorapidity) and calculate the efficiencies dynamically from this information.

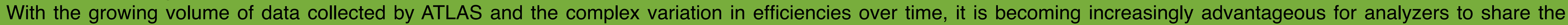

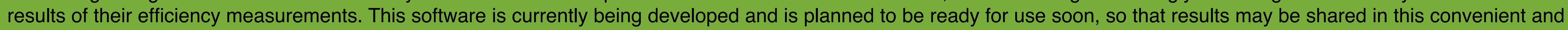
effective way.

\section{References:}

[1] N. Berger et al., "The ATLAS High Level Steering", 2008 J. Phys.: Conf. Ser. 119022013 doi: 10.1088/1742-6596/119/2/022013

[2] T. Eifert et al., "The User Interface for the ATLAS Trigger and Its Use in Physics Studies", CERN, ATL-DAQ-CONF-2008-001
(4) $U E E=\angle E=4$

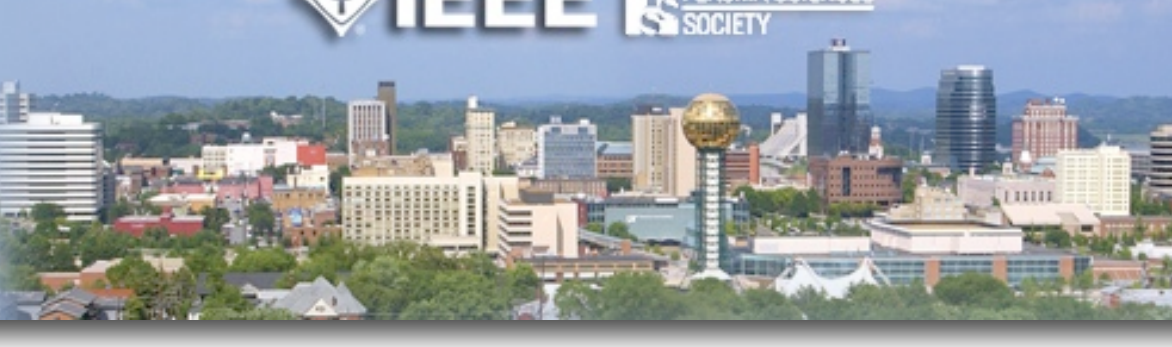

